\title{
Antidepressants and the risk of traumatic brain injury in the elderly: differences between individual agents
}

This article was published in the following Dove Medical Press journal:

Clinical Epidemiology

\author{
Federica Edith Pisa ${ }^{1,2}$ \\ Jonas Reinold' \\ Bianca Kollhorst ${ }^{3}$ \\ Ulrike Haug ${ }^{1,4}$ \\ Tania Schink' \\ 'Clinical Epidemiology, Leibniz \\ Institute for Prevention Research \\ and Epidemiology - BIPS, Bremen, \\ Germany; ${ }^{2}$ Institute of Hygiene and \\ Clinical Epidemiology, University \\ Hospital of Udine, Udine, Italy; \\ ${ }^{3}$ Biometry and Data Management, \\ Leibniz Institute for Prevention \\ Research and Epidemiology - BIPS, \\ Bremen, Germany; ${ }^{4}$ Faculty of Human \\ and Health Sciences, University of \\ Bremen, Bremen, Germany
}

Correspondence: Federica Edith Pisa Department of Clinical Epidemiology, Leibniz Institute for Prevention Research and Epidemiology - BIPS, Achterstrasse 30, 28359 Bremen, Germany

Tel +4942121856869

Fax +4942121856821

Email pisa@leibniz-bips.de
Objective: To determine the association of individual antidepressants (ADs) with the risk of traumatic brain injury (TBI) in the elderly.

Patients and methods: We conducted a case-control study nested in a cohort of new users of ADs aged $\geq 65$ years, identified in the German Pharmacoepidemiological Research Database during 2005-2014. Cases were patients first hospitalized for TBI. Up to 100 controls per case were selected using incidence density sampling. AD use was ascertained the index date based on the supply of last dispensing (adding 150\% of the defined daily doses [DDDs]; in sensitivity analysis, no additional DDDs were considered). We estimated adjusted ORs (aORs) and 95\% CIs using conditional logistic regression.

Results: Among 701,309 cohort members, 16,750 cases were identified and matched to 1,673,320 controls (in both groups: 70.4\% women; median age 80 years). Compared with remote users of the same AD, current users had an aOR (95\% CI) of 1.87 (1.56-2.24) for duloxetine, 1.74 (1.41-2.15) for escitalopram, 1.70 (1.58-1.83) for citalopram, 1.66 (1.40-1.97) for sertraline, $1.64(1.24-2.15)$ for fluoxetine and $1.57(1.20-2.06)$ for paroxetine. The aOR was lower for amitriptyline $(1.45 ; 1.32-1.58)$, trimipramine $(1.17 ; 0.99-1.38)$ and opipramol $(1.11 ; 0.99-1.25)$. Mirtazapine had an aOR of $1.03(0.94-1.12)$. Sensitivity analysis confirmed the findings.

Conclusion: The large variability between individual ADs shows the importance of considering the safety of individual agents rather than focusing on class alone.

Keywords: antidepressants, traumatic brain injury, elderly, health insurance claims databases, pharmacoepidemiology

\section{Introduction}

Traumatic brain injuries (TBIs) are a major health problem in older adults, greatly contributing to short-term mortality ${ }^{1}$ and disability. ${ }^{2}$ Their frequency in the elderly has increased internationally over the last years, with falls being the leading cause in this population. ${ }^{3,4}$ There is thus an urgent need to identify modifiable risk factors, in order to implement effective prevention measures.

Medications are considered one of the most modifiable risk factors, ${ }^{5}$ and use of certain medications, including psychotropic agents, has consistently been associated with an increased risk of falls in the elderly. ${ }^{6,7}$ However, the association of TBI with medications common in the elderly, such as antidepressants (ADs), has hardly been assessed. With a prevalence of use ranging from $9 \%$ to up to one-third in persons aged $\geq 65$ years and increasing with age, ${ }^{8,9}$ ADs are not only a useful therapeutic tool in late-life depression and anxiety disorders but have also been consistently associated 
with increased risk of falls ${ }^{10-12}$ and related injuries. ${ }^{13-17}$ Sedation, dizziness and orthostatic hypotension are among the adverse effects of $\mathrm{AD}$ classes and individual agents, potentially contributing to greater susceptibility to falls and other injuries in the elderly. ${ }^{18}$

To our knowledge, only one study has specifically evaluated the association of TBI with AD use so far. That study, however, was restricted to patients with Alzheimer's disease. ${ }^{19}$ Given that patients with Alzheimer's disease or other dementias are a high-risk group for falls, the results are not generalizable to elderly without dementia. ${ }^{20,21}$ In that study, the risk of TBI was increased by $26 \%$ in users of any AD compared to non-users, and selective serotonin-reuptake inhibitors (SSRIs) were associated with a statistically nonsignificant $17 \%$ increased risk. However, other AD classes and individual ADs have not been evaluated separately, although it is known that the risk profiles generally vary between classes and individual agents. ${ }^{10,22-25}$

Evidence is thus lacking on the risk of TBI associated with $\mathrm{AD}$ use in the elderly beyond selected risk groups, as well as on the variation in risk between $\mathrm{AD}$ classes and individual ADs. To fill these knowledge gaps, we conducted a study in unselected subjects aged $\geq 65$ years who initiated the use of an $\mathrm{AD}$ and aimed to quantify the risk of TBI associated with $\mathrm{AD}$ classes and selected individual ADs.

\section{Patients and methods Ethics and approvals}

In Germany, the utilization of health insurance data for scientific research is regulated by the Code of Social Law. All involved health insurance providers as well as the German Federal (Social) Insurance Office and the Senator for Science, Health and Consumer Protection in Bremen as their responsible authorities evaluated the protocol and approved the use of German Pharmacoepidemiological Research Database (GePaRD) data for this study. GePaRD does not include any identifying patient data. Informed consent for studies based on GePaRD is not required by law. According to the Ethics Committee of the University of Bremen these studies are exempt from institutional review board review (determination 14 November 2013).

\section{Data source}

This study was conducted using the GePaRD. GePaRD is based on claims data from four statutory health insurance providers in Germany and currently includes information on $>20$ million persons who have been insured with one of the participating providers since 2004 or later. In addition to demographic data, GePaRD contains information on drug dispensations (including Anatomical and Therapeutic Code [ATC], defined daily dose [DDD], strength, packaging size, generic and brand name), outpatient and inpatient services and diagnoses. Per data year, there is information on $\sim 17 \%$ of the general population and all geographical regions of Germany are represented. In- and outpatient diagnoses are coded according to the ICD, 10th revision, German Modification (ICD-10-GM). GePaRD data are representative of the German general population with respect to age, sex, region of residence and medication dispensations..$^{26,27}$

The suitability of GePaRD data for pharmacoepidemiological research has been assessed methodologically and by validation studies. ${ }^{28} \mathrm{GePaRD}$ has been used for various types of pharmacoepidemiological studies, such as drug utilization studies in the elderly ${ }^{29,30}$ and studies investigating the risks of medications including ADs. ${ }^{31}$

\section{Study design}

We conducted a case-control study nested in a populationbased cohort of subjects aged $\geq 65$ years who initiated use of an AD (new users) between January 1, 2005 and December 31,2014 (study period). When studying exposures (such as the use of ADs) and other covariates (such as comorbidities) that vary over time, especially in large cohorts, the nested case-control approach represents a computationally efficient alternative to time-to-event analysis of the entire cohort, ${ }^{32}$ while providing valid estimates of the relative risk if the selection of controls is conducted using incidence density sampling and without biasing restrictions. ${ }^{33}$ We only included subjects initiating $\mathrm{AD}$ treatment, excluding prevalent $\mathrm{AD}$ users (new users design) to avoid bias related to the depletion of susceptible prevalent users and under-ascertainment of early adverse effects. ${ }^{34}$ Initiation was defined as receiving a first dispensation for an $\mathrm{AD}$ without having filled one in the preceding 365 days. Subjects entered the cohort at the date of first dispensation (cohort entry). To be eligible, subjects were required 1) to have at least 12 months of continuous enrollment before cohort entry and 2) to have not experienced TBI any time before cohort entry, defined as the absence of TBI-related hospitalization codes any time before cohort entry. Each subject was followed from cohort entry to the date of first hospitalization for TBI, disenrollment from insurance, end of study period or death, whichever occurred first.

Within the person-time at risk, we identified the incident cases of TBI and randomly selected up to 100 controls per case using incidence density sampling within the risk set ${ }^{33}$ with matching on sex, age and length of follow-up. Controls 
were eligible to be selected more than once and could become cases later on during follow-up. ${ }^{33} \mathrm{~A}$ case was defined as any cohort member with a TBI-related hospitalization. Cases were identified by the main discharge codes (Table S1) according to an established definition ${ }^{3}$ adapted to ICD-10-GM. The date of admission was defined as the index date (ID). For controls, the ID was defined as the ID of the corresponding case. Eligible patients hospitalized for any reason at the ID of the case were not at risk of being hospitalized for TBI and thus excluded from the set of potential controls (risk set). ${ }^{3}$

\section{Exposure definition}

Dispensations of ADs were identified through the ATC code N06A and categorized into the following classes (Table S2): tricyclics (TCAs), SSRIs, selective serotonin-noradrenaline reuptake inhibitors (SSNRIs), noradrenergic and specific serotonergic ADs (NASSAs), noradrenaline reuptake inhibitors, monoamine oxidase inhibitors as well as herbal and other ADs (which were not considered for the analysis).

Treatment episodes were defined based on the estimated supply, as the intended treatment duration and daily dose are not registered. To account for lower dosage and compliance in the elderly, supply was estimated as the dispensed amount of DDDs plus $150 \%$ of the DDDs (in sensitivity analysis, no additional DDDs were considered). ${ }^{35,36} \mathrm{~A}$ new dispensation starting during the supply of the previous one marked the start of the new treatment episode. Exposure to ADs was ascertained at ID based on the interval between ID and the end of the most recent prior treatment episode. Based on this interval, cases and controls were classified in the following mutually exclusive categories: 1) current use (supply overlapped ID), 2) recent use (supply ended within 30 days before ID), 3) past use (supply ended 31-90 days before ID) and 4) remote use (supply ended $\geq 91$ days before ID), as shown in Figure S1. This latter category encompassed use of any AD, while the others were defined separately for each class and for selected individual agents. Patients being users of two or more ADs within one exposure category were assigned to the separate category, multiple use. Switching was assessed as a separate variable and defined as current use of an $\mathrm{AD}$ with recent use of an $\mathrm{AD}$ of a different class.

\section{Covariates and potential confounders}

Risk factors of TBI as well as comorbidities, comedications, indicators of lifestyle habits and indicators of overall health status were taken into account as potential confounders and assessed based on data starting from January 1, 2004 to ensure at least 1 year of look-back period for all patients. As risk factors ofTBI, we considered the factors associated with the risk of falls in the elderly, ${ }^{6}$ including multimorbidity, use of multiple medications, some comorbidities (eg, vision disorders) and comedications (eg, antipsychotics, antiepileptics as well as hypnotics and sedatives).

Comorbidities were ascertained based on inpatient and confirmed outpatient diagnoses occurring 1) any time before ID for chronic diseases, 2) within 181 days before and at ID for acute infectious diseases (such as urinary tract infections or influenza, which increase the risk of falling) $)^{37,38}$ and 3) within 365 days before and at ID for comorbidities that are also proxies of indications, such as depression and anxiety disorder. Use of comedications was ascertained based on dispensations occurring 1) any time before ID and 2) within 181 days before and at ID for medications potentially affecting the risk of TBI, such as hypnotics and sedatives. Indicators of lifestyle habits included alcohol abuse, illicit drug use, obesity as well as smoking and were estimated based on related diagnoses and medications any time before ID. As indicators of overall health status, frailty and use of health care, we calculated the Charlson Comorbidity Index according to the method of Quan et al, ${ }^{39}$ the number of different medications dispensed in the last 12 months, nursing home residence (yes/no) and hospitalized time within the 364 days before ID. ICD-10-GM codes used to identify comorbidities are provided in Tables S3 and S4.

\section{Statistical analysis}

Conditional logistic regression was used to estimate matched and confounder-adjusted ORs (aORs), with 95\% CIs, in the current, recent and past use of each AD class, comparing with remote users of any $\mathrm{AD}$, and of the most frequent individual agents, comparing with remote users of the same $\mathrm{AD}$ as reference. In the model specification, we included all potential confounders (full model) to reduce the likelihood of residual confounding. Stratified analyses were performed by age (65-74, 75-84 and $\geq 85$ years), sex and prior diagnosis of dementia and of depression as potential effect modifiers. Depression and dementia were ascertained based on inpatient and confirmed outpatient diagnoses occurring any time before ID. We performed sensitivity analyses 1) estimating supply based on the dispensed DDDs without any addition, 2) using remote users of any AD as reference and 2) using current users of TCAs as reference.

\section{Results}

In the cohort of 701,309 new users contributing 2,916,400 person-years of follow-up, 16,750cases of TBI were identified 
and matched to $1,673,320$ controls. In both groups, $70.4 \%$ were women and the median age was 80 years (IQR 74-85; Table 1). Compared to controls, cases more frequently had a Charlson Comorbidity Index $>2(71.2 \%$ vs $62.5 \%),>5 \%$ hospitalized time $(27.1 \%$ vs $13.4 \%)$, used $\geq 5$ medications $(66.4 \%$ vs $54.7 \%)$, were nursing home residents ( $8.3 \%$ vs $4.0 \%)$ and had conditions potentially affecting the risk of falls. These conditions included dementia (33.2\% vs 18.3\%), Parkinson's disease or other movement disorders $(41.4 \%$ vs $33.7 \%)$, use of antipsychotics (35.1\% vs $25.3 \%$ ) or hypnotics and sedatives (11.8\% vs $9.3 \%)$ and alcohol abuse (7.0\% vs $3.3 \%)$.
In current users, the aOR was 1.86 (95\% CI: 1.78-1.94) for SSRI, 1.84 (1.68-2.01) for SSNRI, 1.69 (1.56-1.83) for multiple ADs and $1.22(1.15-1.29)$ for TCAs, compared to remote users of any AD (Table 2).

Considering current users and comparing with remote users of the same $A D$ as the reference, all individual SSRIs and SSNRIs were associated with an increased aOR of TBI. The aOR ranged from 1.57 (1.20-2.06) for paroxetine to 1.87 (1.56-2.24) for duloxetine, with $>60 \%$ increase for fluoxetine (1.64; 1.24-2.15), sertraline (1.66; 1.40-1.97), citalopram $(1.70 ; 1.58-1.83)$ and escitalopram $(1.74 ; 1.41-2.15)$, as

Table I Characteristics of cases of traumatic brain injury and matched controls

\begin{tabular}{|c|c|c|c|c|c|c|}
\hline \multirow[t]{2}{*}{ Demographics } & \multicolumn{2}{|c|}{ Cases $(n=16,750)$} & \multicolumn{2}{|c|}{$\begin{array}{l}\text { Controls } \\
(n=1,673,320)\end{array}$} & \multirow[b]{2}{*}{$\mathbf{a O R}^{\mathbf{a}}$} & \multirow[b]{2}{*}{$95 \% \mathrm{Cl}^{\mathrm{b}}$} \\
\hline & $\mathbf{n}$ & $\%$ & $\mathbf{n}$ & $\%$ & & \\
\hline \multicolumn{7}{|l|}{ Sex } \\
\hline Women & & & & & - & - \\
\hline Men & & & & & - & - \\
\hline \multicolumn{7}{|l|}{ Age (years) } \\
\hline $65-74$ & 4,492 & 26.8 & 449,145 & 26.8 & - & - \\
\hline $75-84$ & 7,394 & 44.1 & \begin{tabular}{|l|}
739,385 \\
\end{tabular} & 44.2 & - & - \\
\hline$\geq 85$ & 4,864 & 29.0 & 484,790 & 29.0 & - & - \\
\hline Median $\left(25^{\circ} ; 75^{\circ}\right.$ percentile $)$ & 80 & $(74 ; 85)$ & 80 & $(74 ; 85)$ & - & - \\
\hline \multicolumn{7}{|l|}{ Comorbidities } \\
\hline Depressionc $^{c}$ & 8,929 & 53.3 & 795,843 & 47.6 & 1.01 & $(0.98-1.04)$ \\
\hline Anxiety disorders ${ }^{c}$ & 1,964 & 11.7 & 194,354 & 11.6 & 0.90 & $(0.85-0.94)$ \\
\hline Dementia $^{c}$ & 5,562 & 33.2 & 305,519 & 18.3 & 1.75 & $(1.68-1.82)$ \\
\hline Bipolar disorders ${ }^{c}$ & 213 & 1.3 & $\mathrm{II}, 447$ & 0.7 & 1.26 & $(1.09-1.44)$ \\
\hline Schizophrenia $^{c}$ & 223 & 1.3 & 12,158 & 0.7 & 1.14 & $(0.99-1.30)$ \\
\hline Obsessive compulsive disorders ${ }^{c}$ & 49 & 0.3 & 4,425 & 0.3 & 0.97 & $(0.73-1.28$ \\
\hline Parkinson's disease and movement disorders ${ }^{c}$ & 6,942 & 41.4 & 563,184 & 33.7 & 1.14 & $(1.10-1.18$ \\
\hline Delirium $^{c}$ & 488 & 2.9 & 14,732 & 0.9 & 1.51 & $(1.37-1.66)$ \\
\hline Pain $^{d}$ & 15,616 & 93.2 & $1,557,928$ & 93.1 & 0.97 & $(0.91-1.03)$ \\
\hline Myocardial infarction ${ }^{d}$ & 2,167 & 12.9 & $|81,72|$ & 10.9 & 1.02 & $(0.97-1.08)$ \\
\hline Other coronary heart disease $^{d}$ & 8,679 & 51.8 & 805,955 & 48.2 & 1.00 & $(0.96-1.03)$ \\
\hline Atrial fibrillation $^{d}$ & $4,4 I I$ & 26.3 & 320,139 & 19.1 & 1.21 & $(1.16-1.26$ \\
\hline Other arrhythmias and conduction disorders ${ }^{\mathrm{d}}$ & 8,312 & 49.6 & 770,645 & 46.1 & 1.00 & $(0.97-1.04)$ \\
\hline Valvular disorders and endocarditis $^{d}$ & 4,680 & 27.9 & $4 \mid 4,867$ & 24.8 & 1.05 & $(1.01-1.09)$ \\
\hline Pericardial disorders $^{d}$ & 217 & 1.3 & 16,858 & 1.0 & 1.12 & $(0.98-1.28)$ \\
\hline Myocarditis $^{d}$ & 79 & 0.5 & 8,710 & 0.5 & 0.81 & $(0.65-1.01)$ \\
\hline Peripheral vascular disease $^{d}$ & 6,470 & 38.6 & 584,318 & 34.9 & 1.04 & $(1.00-1.07)$ \\
\hline Hypertension $^{d}$ & 14,822 & 88.5 & $1,450,023$ & 86.7 & 1.01 & $(0.95-1.06$ \\
\hline Chronic pulmonary disease $^{d}$ & 8,326 & 49.7 & 830,544 & 49.6 & 0.92 & $(0.89-0.96$ \\
\hline Pulmonary circulation disorders ${ }^{d}$ & 1,294 & 7.7 & 97,524 & 5.8 & 1.11 & $(1.04-1.18)$ \\
\hline $\begin{array}{l}\text { Rheumatoid arthritis, other arthropathies and connective } \\
\text { tissue disorders }\end{array}$ & 4,037 & 24.1 & $40 I, 908$ & 24.0 & 0.98 & $(0.95-1.02)$ \\
\hline Liver insufficiency $^{d}$ & 4,574 & 27.3 & 415,310 & 24.8 & 1.01 & $(0.97-1.04)$ \\
\hline Renal insufficiency ${ }^{d}$ & 3,997 & 23.9 & 330,646 & 19.8 & 1.02 & $(0.98-1.06)$ \\
\hline Cancer ${ }^{\mathrm{d}, \mathrm{e}}$ & 5,193 & 31.0 & 500,400 & 29.9 & 1.00 & $(0.97-1.04)$ \\
\hline Diabetes ${ }^{\mathrm{d}, \mathrm{f}}$ & 6,580 & 39.3 & 592,384 & 35.4 & 1.00 & $(0.96-1.04)$ \\
\hline $\begin{array}{l}\text { Osteoporosis and other diseases of bone density and } \\
\text { structure }^{d}\end{array}$ & 6,681 & 39.9 & 642,462 & 38.4 & 0.97 & $(0.94-1.01)$ \\
\hline Syncope and dizziness $^{d}$ & 9,622 & 57.4 & 786,652 & 47.0 & 1.38 & $(1.34-1.43)$ \\
\hline
\end{tabular}

(Continued) 
Table I (Continued)

\begin{tabular}{|c|c|c|c|c|c|c|}
\hline \multirow[t]{2}{*}{ Demographics } & \multicolumn{2}{|c|}{ Cases $(n=16,750)$} & \multicolumn{2}{|c|}{$\begin{array}{l}\text { Controls } \\
(n=1,673,320)\end{array}$} & \multirow[b]{2}{*}{$\mathbf{a O R}^{\mathbf{a}}$} & \multirow[b]{2}{*}{$95 \% \mathrm{Cl}^{\mathrm{b}}$} \\
\hline & $n$ & $\%$ & $n$ & $\%$ & & \\
\hline Vision disorders $^{d}$ & 13,729 & 82.0 & $1,361,833$ & 81.4 & 1.04 & $(1.00-1.09)$ \\
\hline Dyslipidemia $^{\mathrm{d}}$ & 10,845 & 64.7 & $1,085,092$ & 64.8 & 0.94 & $(0.91-0.97)$ \\
\hline Hip or pelvis fracture ${ }^{d}$ & 2,944 & 17.6 & 217,973 & 13.0 & 0.95 & $(0.91-1.00)$ \\
\hline Other fractures $^{d}$ & 5,907 & 35.3 & 425,188 & 25.4 & 1.38 & $(1.33-1.43)$ \\
\hline Infectious diseases $^{g}$ & 6,642 & 39.7 & 622,183 & 37.2 & 0.97 & $(0.94-1.00)$ \\
\hline \multicolumn{7}{|l|}{ Comedications } \\
\hline Insulin $^{d}$ & $\mathrm{I}, 570$ & 9.4 & 118,763 & 7.1 & 0.98 & $(0.92-1.04)$ \\
\hline Antidiabetic medications $^{d}$ & 3,103 & 18.5 & 263,683 & 15.8 & 1.02 & $(0.97-1.07)$ \\
\hline Antithrombotic medications $^{\mathrm{d}}$ & 10,913 & 65.2 & 917,133 & 54.8 & 1.14 & $(1.10-1.19)$ \\
\hline Cardiac glycosides $^{d}$ & 2,774 & 16.6 & 203,054 & 12.1 & 1.09 & $(1.03-1.14)$ \\
\hline Antiarrhythmic medications ${ }^{d}$ & 895 & 5.3 & $67,07 \mid$ & 4.0 & 1.02 & $(0.95-1.10)$ \\
\hline Other antihypertensive medications ${ }^{d}$ & 1,637 & 9.8 & 158,416 & 9.5 & 0.93 & $(0.88-0.98)$ \\
\hline Diuretics $^{d}$ & 10,424 & 62.2 & 921,438 & 55.1 & 1.02 & $(0.98-1.06)$ \\
\hline Vasodilators $^{d}$ & 5,964 & 35.6 & 572,529 & 34.2 & 0.94 & $(0.91-0.98)$ \\
\hline$\beta$-Adrenergic agonists ${ }^{d}$ & 10,895 & 65.0 & $1,031,895$ & 61.7 & 0.96 & $(0.93-1.00)$ \\
\hline Calcium antagonists $^{\mathrm{d}}$ & 7,680 & 45.9 & 731,923 & 43.7 & 0.97 & $(0.93-1.00)$ \\
\hline ACE inhibitors ${ }^{d}$ & 10,932 & 65.3 & $1,015,792$ & 60.7 & 1.03 & $(0.99-1.07)$ \\
\hline Angiotensin II antagonists $^{d}$ & 5,017 & 30.0 & 500,072 & 29.9 & 0.99 & $(0.95-1.02)$ \\
\hline Lipid-lowering medications $^{d}$ & 7,741 & 46.2 & 719,184 & 43.0 & 1.02 & $(0.99-1.06)$ \\
\hline Hormone therapy ${ }^{d}$ & 4,429 & 26.4 & 456,466 & 27.3 & 0.98 & $(0.94-1.02)$ \\
\hline Glucocorticoids $^{d}$ & 6,189 & 36.9 & 619,758 & 37.0 & 0.97 & $(0.94-1.00)$ \\
\hline 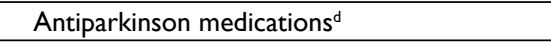 & 2,740 & 16.4 & 211,964 & 12.7 & $\mathrm{I} .03$ & $(0.99-1.08)$ \\
\hline Respiratory medications $^{d}$ & 5,033 & 30.0 & 505,948 & 30.2 & 0.94 & $(0.90-0.98)$ \\
\hline Nonsteroidal anti-inflammatory medications ${ }^{d}$ & 14,232 & 85.0 & $1,409,458$ & 84.2 & 1.04 & $(0.99-1.09)$ \\
\hline Antipsychotics $^{g}$ & 5,880 & 35.1 & 422,562 & 25.3 & 1.06 & $(1.02-1.10)$ \\
\hline Antiepileptics ${ }^{g}$ & 1,954 & 11.7 & 143,021 & 8.5 & 1.07 & $(1.01-1.12)$ \\
\hline Anxiolytics $^{g}$ & 2,059 & 12.3 & 171,987 & 10.3 & 1.03 & $(0.98-1.08)$ \\
\hline Hypnotics and sedatives ${ }^{g}$ & 1,980 & 11.8 & 155,927 & 9.3 & $\mathrm{I} .07$ & $(1.02-1.13)$ \\
\hline Muscle relaxants ${ }^{g}$ & 466 & 2.8 & $5 I, 33 I$ & 3.1 & 0.81 & $(0.74-0.89)$ \\
\hline \multicolumn{7}{|l|}{ Indicators of lifestyle habits } \\
\hline Alcohol abuse & 1,179 & 7.0 & 55,294 & 3.3 & 1.78 & $(1.67-1.90)$ \\
\hline Illicit drug use & 625 & 3.7 & 50,116 & 3.0 & 0.96 & $(0.89-1.04)$ \\
\hline Obesity & 3,886 & 23.2 & 376,683 & 22.5 & 0.93 & $(0.90-0.97)$ \\
\hline Smoking & 792 & 4.7 & 69,861 & 4.2 & 0.95 & $(0.88-1.02)$ \\
\hline \multicolumn{7}{|c|}{ Indicators of health status, frailty and use of health care } \\
\hline Charlson Comorbidity Index $>2^{\mathrm{d}, \mathrm{h}}$ & 11,920 & 71.2 & $1,045,988$ & 62.5 & 1.12 & $(1.07-1.17)$ \\
\hline \multicolumn{7}{|l|}{ Number of medications ${ }^{\mathrm{h}, \mathrm{i}}$} \\
\hline $\mathrm{I}-4$ & 5,296 & 31.6 & 697,888 & 41.7 & 1.10 & $(0.98-1.23)$ \\
\hline $5-8$ & 7,229 & 43.2 & 666,923 & 39.9 & 1.25 & $(I . I I-I .4 I)$ \\
\hline 9 and more & 3,888 & 23.2 & 248,109 & 14.8 & 1.46 & $(1.28-1.66)$ \\
\hline Nursing home residence $e^{c, h}$ & 1,390 & 8.3 & 67,039 & 4.0 & 1.12 & $(1.05-1.19)$ \\
\hline Hospitalized time $>5 \%$ c,h & 4,544 & 27.1 & 224,770 & 13.4 & 1.72 & $(1.65-1.79)$ \\
\hline
\end{tabular}

Notes: ${ }^{M}$ Model adjusted for myocardial infarction, other coronary heart disease, atrial fibrillation, other cardiac arrhythmias and conduction disorders, valvular disorders and endocarditis, pericardial disorders, myocarditis, peripheral vascular disease, hypertension, dementia, chronic pulmonary disease, pulmonary circulation disorders, rheumatic arthritis/collagen vascular disease, liver disease, Parkinson's disease, other extrapyramidal and movement disorders, depression, diabetes, renal failure, cancer (except malignant neoplasm of the skin), obesity, alcohol abuse, drug abuse, osteoporosis and other disorders of bone density and structure, syncope, dizziness and tendency to fall, vision disorders, dyslipidemia, bipolar disorders, schizophrenia, smoking, anxiety disorders, obsessive compulsive disorders, other movement disorders, pain, infectious diseases, delirium, use of insulin, antidiabetic medications, antithrombotic medications, cardiac glycosides, antiarrhythmic medications, other antihypertensive medications, diuretics, vasodilators, $\beta$-adrenergic agonists, calcium antagonists, ACE inhibitors, angiotensin II antagonists, lipid-lowering medications, hormone therapy, glucocorticoids, antiparkinson medications, respiratory medications, nonsteroidal anti-inflammatory medications, antipsychotics, antiepileptic medications, anxiolytics, hypnotics and sedatives, muscle relaxants, number of medications $\left(I-4,5-8,9\right.$ and more), hospitalized time $(\leq 5 \%,>5 \%)$, Charlson Comorbidity Index $(\leq 2,>2)$ and nursing home residence. ${ }^{5} 95 \%$ $\mathrm{Cl}$. 'Assessed within 365 days before the index date and at the index date. ${ }^{\mathrm{d} A s s e s s e d}$ any time before the index date. ${ }^{\mathrm{E}} \mathrm{Except}$ malignant neoplasm of the skin. Includes both type I and type 2 diabetes. ${ }^{8}$ Assessed within I8I days before and at the index date. ${ }^{h}$ Cutoffs were defined as follows: for Charlson Comorbidity Index the first tertile among controls, for number of medications prior studies, for hospitalized time the first decile among controls. Medication of different therapeutic classes.

Abbreviations: ACE, angiotensin converting enzyme; aOR, adjusted OR. 
Table 2 OR with $95 \% \mathrm{Cl}$ of traumatic brain injury in current, recent and past users of ADs compared with remote users of any AD

\begin{tabular}{|c|c|c|c|c|c|c|}
\hline & \multicolumn{2}{|c|}{ Cases $(n=16,750)$} & \multicolumn{2}{|c|}{ Controls $(n=1,673,320)$} & \multirow{2}{*}{$\begin{array}{l}\text { Matched OR } \\
(95 \% \mathrm{Cl})\end{array}$} & \multirow{2}{*}{$\begin{array}{l}\text { Adjusted OR } \\
(95 \% \mathrm{Cl})\end{array}$} \\
\hline & $\mathbf{n}$ & $\%$ & $\mathbf{n}$ & $\%$ & & \\
\hline Remote use of any $A D^{c}$ & 8,432 & 50.3 & $1,004,327$ & 60.0 & $1.00(-)$ & $1.00(-)$ \\
\hline \multicolumn{7}{|l|}{ TCA } \\
\hline Current use & 1,620 & 9.7 & 172,230 & 10.3 & $1.30(1.23-1.38)$ & $1.22(1.15-1.29)$ \\
\hline Recent use & 394 & 2.4 & 48,554 & 2.9 & $1.14(1.03-1.26)$ & $1.11(1.00-1.23)$ \\
\hline Past use & 422 & 2.5 & 60,234 & 3.6 & $0.97(0.88-1.08)$ & $0.98(0.88-1.08)$ \\
\hline \multicolumn{7}{|l|}{ SSRI } \\
\hline Current use & 3,735 & 22.3 & 205,603 & 12.3 & $2.52(2.4 I-2.62)$ & $1.86(1.78-1.94)$ \\
\hline Recent use & 104 & 0.6 & 9,101 & 0.5 & $1.59(1.31-1.94)$ & $1.27(1.05-1.55)$ \\
\hline Past use & 158 & 0.9 & 14,429 & 0.9 & I.5I (I.29-I.77) & $1.23(1.04-1.44)$ \\
\hline \multicolumn{7}{|l|}{ NASSA } \\
\hline Current use & 1,273 & 7.6 & 115,607 & 6.9 & $1.53(1.44-1.63)$ & $1.12(1.05-1.20)$ \\
\hline Recent use & 104 & 0.6 & 9,875 & 0.6 & $1.48(1.22-1.80)$ & $1.22(1.01-1.49)$ \\
\hline Past use & 131 & 0.8 & 13,266 & 0.8 & $1.37(1.15-1.63)$ & $1.15(0.96-1.37)$ \\
\hline \multicolumn{7}{|l|}{ SSNRI } \\
\hline Current use & 575 & 3.4 & 31,335 & 1.9 & $2.48(2.27-2.70)$ & $1.84(1.68-2.0 \mathrm{I})$ \\
\hline Recent use & 24 & 0.1 & 2,110 & 0.1 & $1.58(1.06-2.37)$ & $1.27(0.85-1.90)$ \\
\hline Past use & 26 & 0.2 & 2,956 & 0.2 & $1.21(0.82-1.78)$ & $0.97(0.66-1.43)$ \\
\hline \multicolumn{7}{|l|}{ MAO } \\
\hline Current use & 13 & 0.1 & 1,734 & 0.1 & $1.04(0.60-1.79)$ & $0.80(0.46-1.38)$ \\
\hline Recent use & 3 & $<0.1$ & 107 & $<0.1$ & $3.95(1.26-12.37)$ & $3.27(1.03-10.37)$ \\
\hline Past use & 2 & $<0.1$ & 167 & $<0.1$ & $1.66(0.4 \mathrm{I}-6.70)$ & $1.35(0.33-5.46)$ \\
\hline \multicolumn{7}{|l|}{ NARI } \\
\hline Current use & 19 & 0.1 & $\mathrm{I}, 207$ & 0.1 & $2.20(1.40-3.47)$ & $1.55(0.98-2.45)$ \\
\hline Recent use & 2 & $<0.1$ & 138 & $<0.1$ & $2.04(0.50-8.22)$ & $1.31(0.32-5.32)$ \\
\hline Past use & I & $<0.1$ & 188 & $<0.1$ & $0.74(0.10-5.29)$ & $0.55(0.08-3.96)$ \\
\hline \multicolumn{7}{|l|}{ Other Ads } \\
\hline Current use & 77 & 0.5 & 5,153 & 0.3 & $2.05(1.63-2.57)$ & $1.45(1.15-1.82)$ \\
\hline Recent use & 8 & $<0.1$ & 716 & $<0.1$ & $1.57(0.78-3.14)$ & $1.07(0.53-2.16)$ \\
\hline Past use & 13 & 0.1 & 918 & 0.1 & $1.94(1.12-3.36)$ & $\mathrm{I} .4(0.8 \mathrm{I}-2.42)$ \\
\hline \multicolumn{7}{|l|}{ Multiple AD } \\
\hline Current use & $\mathrm{I}, 005$ & 6.0 & 52,313 & 3.1 & $2.58(2.42-2.76)$ & $1.69(1.56-1.83)$ \\
\hline Recent use & 7 & $<0.1$ & 603 & $<0.1$ & $1.59(0.75-3.35)$ & $1.17(0.55-2.47)$ \\
\hline Past use & 16 & 0.1 & 1,462 & 0.1 & $1.49(0.91-2.43)$ & $1.19(0.73-1.96)$ \\
\hline Current switchers $^{d}$ & 556 & 3.3 & 28,675 & 1.7 & $1.97(1.81-2.15)$ & $1.08(0.98-1.19)$ \\
\hline
\end{tabular}

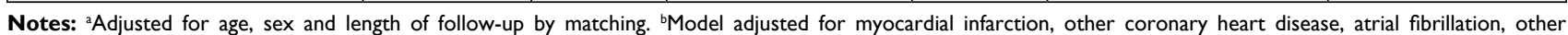
cardiac arrhythmias and conduction disorders, valvular disorders and endocarditis, pericardial disorders, myocarditis, peripheral vascular disease, hypertension, dementia, chronic pulmonary disease, pulmonary circulation disorders, rheumatic arthritis/collagen vascular disease, liver disease, Parkinson's disease, other extrapyramidal and movement disorders, depression, diabetes, renal failure, cancer (except malignant neoplasm of the skin), obesity, alcohol abuse, drug abuse, osteoporosis and other disorders of bone density and structure, syncope, dizziness and tendency to fall, vision disorders, dyslipidemia, bipolar disorders, schizophrenia, smoking, anxiety disorders, obsessive compulsive disorders, other movement disorders, pain, infectious diseases, delirium, use of insulin, antidiabetic medications, antithrombotic medications, cardiac glycosides, antiarrhythmic medications, other antihypertensive medications, diuretics, vasodilators, $\beta$-adrenergic agonists, calcium antagonists, ACE inhibitors, angiotensin II antagonists, lipid-lowering medications, hormone therapy, glucocorticoids, antiparkinson medications, respiratory medications, nonsteroidal anti-inflammatory medications, antipsychotics, antiepileptic medications, anxiolytics, hypnotics and sedatives, muscle relaxants, number of medications (I-4, 5-8, 9 and more), hospitalized time ( $\leq 5 \%,>5 \%)$, Charlson Comorbidity Index $(\leq 2,>2)$, nursing home residence. 'Reference category. ${ }^{\circ}$ Current switching: current use of one $A D$ class with recent use of a different $A D$ class. Abbreviations: ACE, angiotensin converting enzyme; AD, antidepressant; MAO, monoamine oxidase inhibitor; NARI, noradrenaline reuptake inhibitor; NASSA, noradrenergic and specific serotonergic antidepressant; SSNRI, selective serotonin-noradrenaline reuptake inhibitor; SSRI, selective serotonin reuptake inhibitor; TCA, tricyclic.

shown in Table 3. Among TCAs, the aOR was increased only for opipramol $(1.11 ; 0.99-1.25)$, trimipramine $(1.17$; $0.99-1.38)$ and amitriptyline $(1.45 ; 1.32-1.58)$. Mirtazapine had an aOR of 1.03 (0.94-1.12). Recent users of fluoxetine (2.18; 1.01-4.73) and amitriptyline (1.38; 1.17-1.62) had statistically significant increased risk. Using remote users of any $\mathrm{AD}$ as the reference, the increase in aOR was statistically significant also in recent and past users of citalopram (1.33; $1.07-1.66$ and $1.26 ; 1.05-1.51$, respectively) and in recent users of mirtazapine $(1.22 ; 1.00-1.48)$.

Patients with and without dementia had a similar pattern of aOR for all ADs (Figure 1).

Sensitivity analysis using the DDDs without any addition to estimate supply confirmed the results in current users of all 
Table 3 OR with $95 \% \mathrm{Cl}$ of traumatic brain injury in current, recent and past users of individual AD compared with remote users of the same individual $A D$ or with remote users of any $A D$

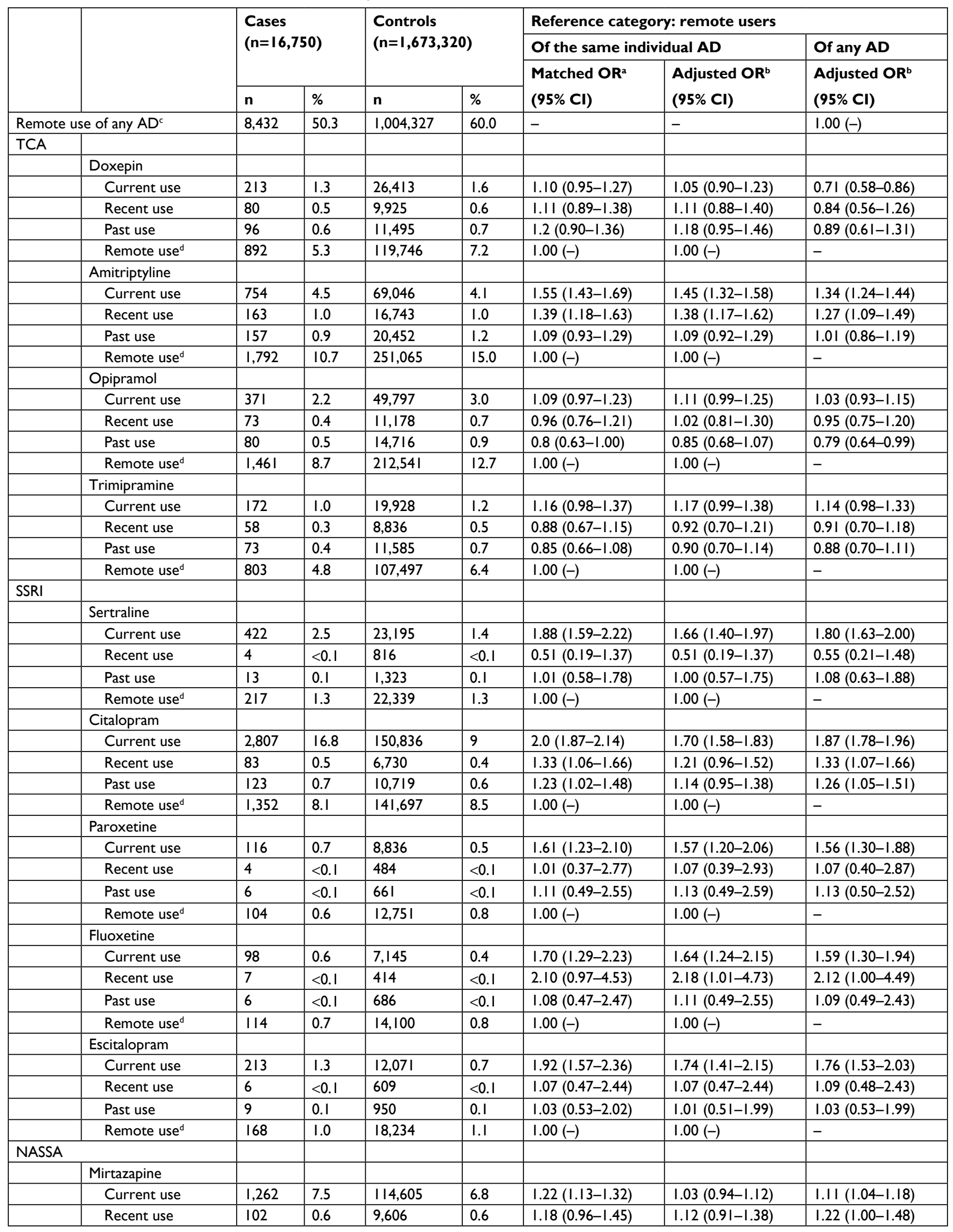

(Continued) 
Table 3 (Continued)

\begin{tabular}{|c|c|c|c|c|c|c|c|c|}
\hline & & \multirow{2}{*}{\multicolumn{2}{|c|}{$\begin{array}{l}\text { Cases } \\
(n=16,750)\end{array}$}} & \multirow{2}{*}{\multicolumn{2}{|c|}{$\begin{array}{l}\text { Controls } \\
(n=1,673,320)\end{array}$}} & \multicolumn{3}{|c|}{ Reference category: remote users } \\
\hline & & & & & & \multicolumn{2}{|c|}{ Of the same individual AD } & \multirow{3}{*}{$\begin{array}{l}\text { Of any AD } \\
\text { Adjusted OR } \\
(95 \% \mathrm{Cl}) \\
\end{array}$} \\
\hline & & & & & & \multirow{2}{*}{$\begin{array}{l}\text { Matched OR } \\
(95 \% \mathrm{Cl})\end{array}$} & \multirow{2}{*}{$\begin{array}{l}\text { Adjusted OR } \\
(95 \% \mathrm{Cl})\end{array}$} & \\
\hline & & $\mathbf{n}$ & $\%$ & $\mathbf{n}$ & $\%$ & & & \\
\hline & Past use & 128 & 0.8 & 12,944 & 0.8 & $1.10(0.91-1.32)$ & $1.05(0.87-1.26)$ & $1.13(0.95-1.35)$ \\
\hline & Remote use $^{d}$ & $|, 35|$ & 8.1 & 149,402 & 8.9 & $\mathrm{I} .00(-)$ & $1.00(-)$ & \\
\hline \multicolumn{9}{|l|}{ SSNRI } \\
\hline & Duloxetine & & & & & & & \\
\hline & Current use & 267 & 1.6 & 14,857 & 0.9 & $2.14(1.79-2.55)$ & $1.87(1.56-2.24)$ & $1.81(1.59-2.05)$ \\
\hline & Recent use & 11 & 0.1 & $\mathrm{I}, \mathrm{I} 70$ & 0.1 & $1.12(0.6 \mathrm{I}-2.06)$ & $\mathrm{I} .12(0.6 \mathrm{I}-2.06)$ & $\mathrm{I} .08(0.60-\mathrm{I} .97)$ \\
\hline & Past use & 17 & 0.1 & $\mathrm{I}, 726$ & 0.1 & $\mathrm{I} .17(0.7 \mathrm{I}-\mathrm{I} .92)$ & $1.12(0.68-1.85)$ & $1.09(0.67-1.76)$ \\
\hline & Remote use $^{d}$ & 237 & 1.4 & 28,123 & 1.7 & $1.00(-)$ & $1.00(-)$ & - \\
\hline & Venlafaxine & & & & & & & \\
\hline & Current use & 302 & 1.8 & 16,335 & 1.0 & 2.01 (I.66-2.42) & $1.76(1.45-2.15)$ & $1.81(1.6 \mathrm{I}-2.04)$ \\
\hline & Recent use & 13 & 0.1 & 939 & 0.1 & $1.5(0.85-2.65)$ & I.4I (0.80-2.50) & $\mathrm{I} .45(0.84-2.5 \mathrm{I})$ \\
\hline & Past use & 9 & 0.1 & 1,222 & 0.1 & $0.8(0.4 I-I .57)$ & $0.76(0.39-1.50)$ & $0.78(0.4|-| .5 I)$ \\
\hline & Remote use $^{d}$ & 171 & 1.0 & 18,576 & I.I & $1.00(-)$ & $1.00(-)$ & - \\
\hline
\end{tabular}

Notes: ${ }^{a}$ Adjusted for age, sex and length of follow-up by matching. 'Model adjusted for myocardial infarction, other coronary heart disease, atrial fibrillation, other cardiac arrhythmias and conduction disorders, valvular disorders and endocarditis, pericardial disorders, myocarditis, peripheral vascular disease, hypertension, dementia, chronic pulmonary disease, pulmonary circulation disorders, rheumatic arthritis/collagen vascular disease, liver disease, Parkinson's disease, other extrapyramidal and movement disorders, depression, diabetes, renal failure, cancer (except malignant neoplasm of the skin), obesity, alcohol abuse, drug abuse, osteoporosis and other disorders of bone density and structure, syncope, dizziness and tendency to fall, vision disorders, dyslipidemia, bipolar disorders, schizophrenia, smoking, anxiety disorders, obsessive compulsive disorders, other movement disorders, pain, infectious diseases, delirium, use of insulin, antidiabetic medications, antithrombotic medications, cardiac glycosides, antiarrhythmic medications, other antihypertensive medications, diuretics, vasodilators, $\beta$-adrenergic agonists, calcium antagonists, ACE inhibitors, angiotensin II antagonists, lipid-lowering medications, hormone therapy, glucocorticoids, antiparkinson medications, respiratory medications, nonsteroidal anti-inflammatory medications, antipsychotics, antiepileptic medications, anxiolytics, hypnotics and sedatives, muscle relaxants, number of medications ( $1-4,5-8,9$ and more), hospitalized time ( $\leq 5 \%$, $>5 \%)$, Charlson Comorbidity Index $(\leq 2,>2)$, nursing home residence. 'Reference category remote users of any $A D$. ${ }^{d}$ Reference category remote users of the same $A D$.

Abbreviations: ACE, angiotensin converting enzyme; AD, antidepressant; MAO, monoamine oxidase inhibitor; NARI, noradrenaline reuptake inhibitor; NASSA, noradrenergic and specific serotonergic antidepressant; SSNRI, selective serotonin-noradrenaline reuptake inhibitor; SSRI, selective serotonin reuptake inhibitor; TCA, tricyclic.

ADs (Table S5). Compared to current users of TCAs, current users of SSRIs had an aOR of $1.52(1.43-1.62)$ and current users of SSNRIs had an aOR of 1.51 (1.37-1.66), as shown in Table S6. Results were similar in all age groups (Figure S2), between men and women (Figure $\mathrm{S} 3$ ) and when the analysis was restricted to patients with depression (Figure S4).

\section{Discussion}

To our knowledge, this is the first study assessing the risk of TBI among users of ADs in an unselected populationbased cohort of older adults. Given our large sample size of $>700,000$ new users, we were able to assess the risk for individual ADs. Taking into account a wide range of comorbidities, use of comedications and other potential confounding factors, we found that current users of duloxetine had $>80 \%$ increased risk of TBI and that fluoxetine, sertraline, citalopram and escitalopram had $>60 \%$ increased risk, compared to remote users of the same $\mathrm{AD}$. The increase in risk was lower for current users of paroxetine (57\%), amitriptyline (45\%), trimipramine (17\%) and opipramol (11\%). All examined individual SSRIs had a higher risk than the individual TCAs. On the one hand, this is unexpected, given that both classes may induce adverse effects such as sedation and dizziness ${ }^{18}$ but on the other hand, also for hip fractures, another fall-related outcome, studies consistently found a stronger association for SSRIs than for TCAs. ${ }^{24,40}$ Potential mechanism explaining this difference should be explored. The large variability between individual ADs observed in our study shows the importance of considering the safety of individual agents rather than focusing on $\mathrm{AD}$ class alone.

So far, the association between TBI and AD use has been investigated in only one study that was restricted to a high-risk group, that is, patients with Alzheimer's disease. ${ }^{19}$ In that study, the risk in current users of SSRIs was $17 \%$, which was lower than in our study and did not reach statistical significance due to the limited number of TBIs among SSRIs users. The difference in risk estimates may be explained by differences in the proportion of individual ADs included in the SSRI class, reflecting study-specific prescribing patterns.

Due to the lack of studies focusing on the association between TBI and individual agents, our results can only be 


\begin{tabular}{|c|c|}
\hline & $\begin{array}{c}\text { Dementia: yes } \\
\text { aOR } 95 \% \mathrm{Cl}\end{array}$ \\
\hline & Doxepin \\
\hline Current use & $0.99(0.72-1.36)$ \\
\hline Recent use & $0.89(0.53-1.51)$ \\
\hline Past use & $1.45(0.93-2.26)$ \\
\hline \multicolumn{2}{|l|}{ Amitriptyline } \\
\hline Current use & $1.38(1.13-1.67)$ \\
\hline Recent use & $1.50(1.06-2.13)$ \\
\hline \multirow{2}{*}{\multicolumn{2}{|c|}{ Opipramol }} \\
\hline & \\
\hline Current use & $1.05(0.83-1.34)$ \\
\hline Recent use & $1.23(0.76-2.01)$ \\
\hline \multicolumn{2}{|l|}{$\begin{array}{l}\text { Past use } \\
\text { Trimipramine }\end{array}$} \\
\hline Current use & $1.12(0.81-1.53)$ \\
\hline Recent use & $0.78(0.44-1.36)$ \\
\hline Past use & $0.87(0.53-1.44)$ \\
\hline \\
\hline \multicolumn{2}{|l|}{ Sertraline } \\
\hline Current use & $1.43(1.09-1.86)$ \\
\hline Recent use & - \\
\hline \multirow{2}{*}{\multicolumn{2}{|c|}{ Citalopram }} \\
\hline & \\
\hline Current use & $1.58(1.40-1.78)$ \\
\hline Recent use & $1.36(0.96-1.92)$ \\
\hline Past use & $1.03(0.75-1.41)$ \\
\hline \multicolumn{2}{|l|}{ Paroxetine } \\
\hline Current use & $1.06(0.63-1.79)$ \\
\hline Recent use & $0.75(0.09-6.06)$ \\
\hline Past use & $0.87(0.07-4.35)$ \\
\hline \multicolumn{2}{|l|}{ Fluoxetine } \\
\hline Current use & $1.63(1.00-2.66)$ \\
\hline Recent use & $2.13(0.46-9.82)$ \\
\hline Past use & $0.89(0.13-7.55)$ \\
\hline \multicolumn{2}{|l|}{ Escitalopram } \\
\hline Current use & $1.34(0.95-1.89)$ \\
\hline Recent use & $0.43(0.06-3.18)$ \\
\hline Past use & $1.22(0.43-3.48)$ \\
\hline \multirow{2}{*}{\multicolumn{2}{|c|}{$\begin{array}{l}\text { NASSA } \\
\text { Mirtazapine }\end{array}$}} \\
\hline & \\
\hline Current use & $0.98(0.85-1.13)$ \\
\hline Recent use & $1.16(0.82-1.63)$ \\
\hline Past use & $1.07(0.78-1.47)$ \\
\hline \multicolumn{2}{|l|}{ SSNRI } \\
\hline \multicolumn{2}{|l|}{ Duloxetine } \\
\hline Current use & $1.51(1.05-2.1$ \\
\hline Recent use & $0.38(0.09-1.6$ \\
\hline \multirow{2}{*}{\multicolumn{2}{|c|}{ Venlafaxine }} \\
\hline & \\
\hline Current use & $1.32(0.92-1.89)$ \\
\hline Recent use & $1.90(0.77-4.73)$ \\
\hline Past use & - \\
\hline
\end{tabular}

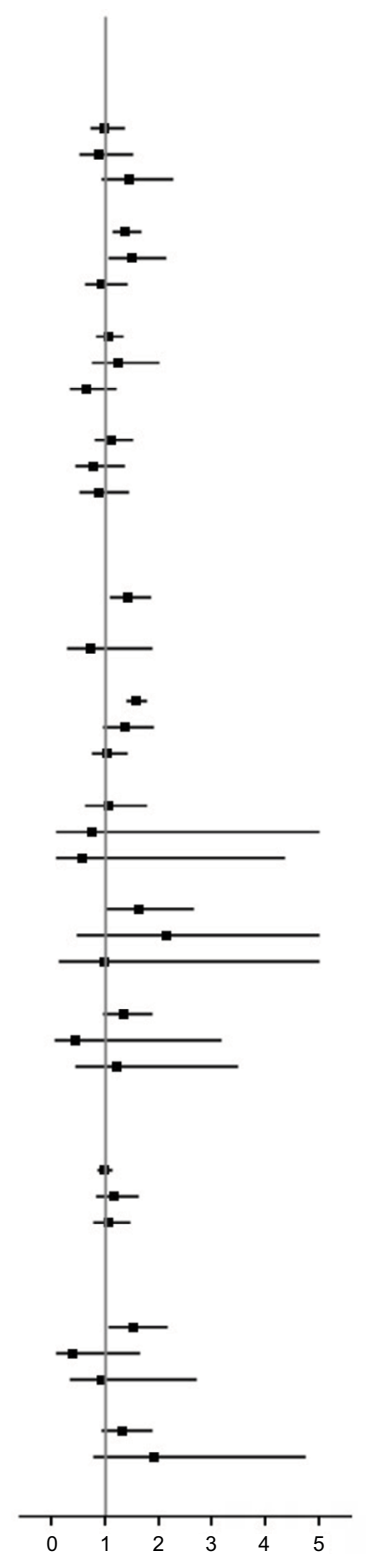

Dementia: no

aOR 95\% Cl

$1.05(0.88-1.25)$

$1.16(0.89-1.50)$

$1.11(0.87-1.43)$

$1.44(1.30-1.60)$

$1.31(1.09-1.58)$

$1.14(0.95-1.37)$

$1.09(0.95-1.25)$

$0.98(0.74-1.30)$

$0.93(0.72-1.19)$

$1.14(0.93-1.39)$

$1.00(0.73-1.36)$

$0.91(0.69-1.21)$

$1.79(1.43-2.25)$

$0.98(0.36-2.67)$

$1.08(0.52-2.22)$

1.80 (1.64-1.97)

$1.12(0.82-1.51)$

$1.20(0.94-1.53)$

$1.75(1.27-2.41)$

$1.09(0.34-3.48)$

$1.28(0.51-3.20)$

$1.59(1.13-2.24)$

$2.51(1.00-6.28)$

$1.33(0.53-3.32)$

$1.96(1.50-2.57)$

$1.45(0.58-3.61)$

$0.99(0.40-2.45)$

$1.00(0.90-1.12)$

$1.05(0.80-1.36)$

$0.99(0.78-1.25)$

$1.92(1.55-2.38)$

$1.37(0.69-2.69)$

$1.15(0.65-2.04)$

$2.00(1.58-2.53)$

$1.18(0.54-2.55)$

$1.03(0.50-2.12)$

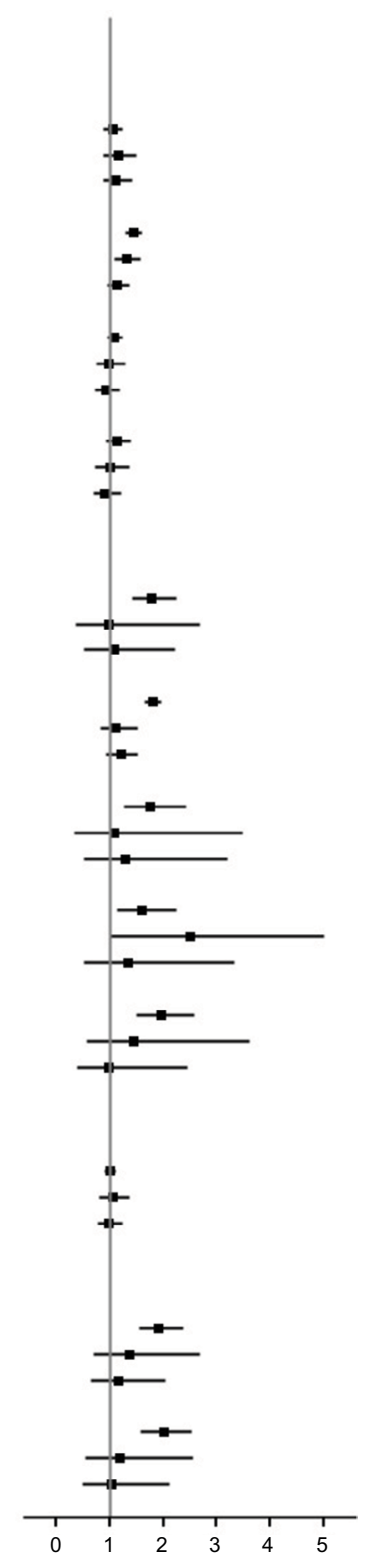

Figure I aOR with $95 \% \mathrm{Cl}$ of traumatic brain injury in users of antidepressants compared with remote users of any $\mathrm{AD}$, by diagnosed dementia. Abbreviations: AD, antidepressant; aOR, adjusted OR; NASSA, noradrenergic and specific serotonergic antidepressant; SSNRI, selective serotonin-noradrenaline reuptake inhibitor; SSRI, selective serotonin reuptake inhibitor; TCA, tricyclic.

indirectly compared with studies investigating falls as an outcome, the main cause of TBI in the elderly. In one large cohort of elderly with depression based on a general practice care database in the UK, the risk of falls was increased between $60 \%$ and $80 \%$ for citalopram, escitalopram, sertraline, fluoxetine and venlafaxine, between $30 \%$ and $50 \%$ for paroxetine and amitriptyline and by $20 \%$ for mirtazapine. ${ }^{25}$ In another cohort of French community-dwelling elderly based on inperson interview and clinical examination restricted to SSRIs, the risk of falls was twofold increased for citalopram, by $50 \%$ for sertraline and paroxetine and by $38 \%$ for fluoxetine. ${ }^{41} \mathrm{We}$ could not compare our findings regarding duloxetine because both studies did not evaluate this AD.

In the interpretation of our results, limitations due to the nature of secondary data have to be considered, including lack of direct information on the duration of treatment or direct clinical measures of certain patient characteristics such as frailty or cognitive status. We used elaborate meth- 
ods to overcome these limitations as much as possible, as addressed and discussed in the following paragraphs for the various aspects.

In our study, the risk remained elevated shortly (30 days) after the end of supply for fluoxetine and amitriptyline. Similarly, in a large multidatabase study, the risk of hip fractures remained elevated within 60 days after the end of AD treatment. ${ }^{16}$ Our finding may partially be explained by residual misclassification of exposure, resulting from estimating treatment episodes without considering overlapping supply from prior dispensings and variations of dose by indication and during the treatment (eg, titration beyond the starting dose). As the results did not change substantially in sensitivity analysis assuming no additional dose, relevant misclassification of exposure is unlikely. Moreover, the strength of our study is the ascertainment of exposure based on dispensations. Contrary to prescriptions or information extracted from medical documentation, dispensations reflect medications actually redeemed at the pharmacy level.

An alternative explanation is that the risk was actually increased over the first weeks after treatment end because of persisting medication effects or as a consequence of discontinuation symptoms such as dizziness, tremors or sleep disturbances.

We defined the outcome as TBI leading to hospitalization, thus focusing on clinically relevant events. Milder events not leading to hospitalization have not been captured, but their number should be low since elderly patients also with milder TBI are commonly evaluated in the hospital. In Germany, hospital main discharge diagnoses are considered to have a high validity since they are based on all information relevant to diagnosis (including laboratory tests and imaging results) during the in-hospital stay and are subject to regular inspection. TBI is generally accurately diagnosed, since imaging is indicated in all adult trauma patients with presentation suggestive of TBI (including but not limited to loss or disturbances of consciousness, signs of skull fracture, cerebrospinal fluid leak), but recommended also in dubious cases, who are kept under observation. ${ }^{42}$ Therefore, misclassification of the outcome should be minimal. Moreover, hospital main discharge diagnoses are based on all information relevant to the diagnosis (including laboratory tests and imaging results) produced during the in-hospital stay. Out-of-hospital deaths due to TBI were not included since causes of death are not available in GePaRD. However, age-adjusted rates of mortality for TBI are much lower than those of hospital discharge rates for TBI in Europe and in Germany specifically, ${ }^{4}$ and the resulting potential bias was most likely non-differential with respect to use of AD. As the ICD-10-GM classification does not include codes for external causes of injuries, we could not perform sensitivity analyses restricting to events coded as fall-related TBIs. Falls are by large the leading cause of TBI in the elderly, while causes other than falls (such as being struck by or against an object and motor vehicle crashes) are much rarer in this population ${ }^{3,4}$ and most likely occur equally with respect to $\mathrm{AD}$ use. Thus, the relative risk should not be biased. Moreover, external cause coding has a very low accuracy. ${ }^{43}$

We extensively adjusted for several potential confounders, including proxies for indications, and the adjustment slightly lowered the point estimates, suggesting that confounding effects were controlled for. Nevertheless, residual confounding, particularly by indication, may have influenced the results. SSRIs are considered the first-line therapy for late-life depression due to their superior safety profile over TCAs, and may have been preferentially prescribed to patients with multiple comorbidities, who are at higher risk of falls. ${ }^{6}$ ADs in the elderly are used for a wide spectrum of indications, which differs considerably between individual agents.

However, the results in the more homogeneous group of patients with depression were comparable to the overall results; thus, residual confounding by indication likely was small, if there was any. Moreover, when we compared with subjects currently treated with TCAs (active treatment comparator), current users of SSRIs and SSNRIs had lower, but still statistically significant increased risks. We compared subjects currently treated with ADs with subjects who used any ADs in the past but were not currently treated. If the indication for treatment independently increases the risk of TBI, the excess risk may have been overestimated (indication bias). However, the analysis comparing with subjects who used the same AD in the past confirmed the results, except for a lack of association in current users of mirtazapine and $a \geq 10$ percentage points difference in current users of sertraline (with risk slightly decreased) and amitriptyline (with risk slightly increased).

Between-person comparisons do not account for confounding due to patient-specific characteristics that are constant over time, such as habitual physical activity, permanent disabilities or constant regular use of nonprescription medications. We thus assessed the feasibility of a casecrossover analysis. Based on within-person comparison, the case-crossover design effectively addresses time-invariant confounders and provides valid estimates of risk when the exposure is transient and the outcome is acute. ${ }^{44,45}$ Although we used multiple control time windows, the very low number of subjects with discordant exposure in the case and control 
time windows hindered meaningful and precise risk estimates to be obtained. In this cohort, ADs were mostly taken chronically or over long periods; thus, the requirement for a transient exposure was met only in the small subgroup of intermittent or non-adherent users, which may substantially differ from continuous users. Thus, the resulting estimates were not only highly imprecise, but also flawed by selection bias.

Residual confounding due to factors unmeasured or not adequately measured in secondary data may persist. These factors include lifestyle habits, frailty, cognitive and functional impairment, history of falls and severity of depression. Among lifestyle habits, alcohol abuse, in particular, may be associated with the risk of TBI. We estimated the indicators of lifestyle habits based on related diagnoses and medications; therefore, we accounted for alcohol abuse with impact on patient health, but likely not for less-severe or more recent abuse. We adjusted for several indicators of frailty independently, such as nursing home residency, comorbidities and number of medications, to account for multiple dimensions of frailty with different effects on fall risk. Moreover, we adjusted for prior hip and other fractures as indicators of (at least the most severe) falls and also for medications potentially affecting the risk of falls assessed shortly before and at ID to better approximate concurrent use.

\section{Conclusion}

In this large cohort of unselected elderly new users of ADs, the risk of TBI was increased in current users of most ADs compared to remote user of any AD. Taking into account a wide range of comorbidities, use of comedications and other potential confounding factors, we observed differences between individual agents. The increase in risk was higher in current users of duloxetine ( $>80 \%$ ), fluoxetine, sertraline, citalopram and escitalopram $(>60 \%)$, while it was lower in current users of paroxetine (57\%), amitriptyline (45\%), trimipramine $(17 \%)$ and opipramol $(11 \%)$. These results were consistent in subjects with and without depression or dementia, in men and women and across all age groups.

While all examined individual SSRIs had a higher risk than the individual TCAs, the large variability between individual ADs shows the importance of considering the safety of individual agents rather than focusing on class alone.

\section{Acknowledgments}

The initial phase of this study was financed by the German Federal Institute for Drugs and Medical Devices (Bundesinstitut für Arzneimittel und Medizinprodukte). The publication of this article was funded by the Open Access Fund of the Leibniz Association. The funders had no role in study design, data collection and analysis, decision to publish or preparation of the manuscript. An abstract of part of this paper was presented at the 14th International Congress of the European Geriatric Medicine Society, October 10-12, 2018, Berlin, Germany, as an abstract presentation with interim and partial findings, and was published in Abstracts of the 14th International Congress of the European Geriatric Medicine Society; Eur Geriatr Med. 2018;9(Suppl 1):1-367 (https://doi.org/10.1007/s41999-018-0097-4). An abstract of part of this paper was also presented at the 34th International Conference on Pharmacoepidemiology and Therapeutic Risk Management, August 22-26, 2018, Prague, Czech Republic, as an oral presentation with interim and partial findings, and was published in Pharmacoepidemiol Drug Saf; 2018;27(S2):1-2 (https:// doi.org/10.1002/pds.4628).

\section{Disclosure}

The authors report no conflicts of interest in this work.

\section{References}

1. McIntyre A, Mehta S, Aubut J, Dijkers M, Teasell RW. Mortality among older adults after a traumatic brain injury: a meta-analysis. Brain Inj. 2013;27(1):31-40.

2. Zaloshnja E, Miller T, Langlois JA, Selassie AW. Prevalence of long-term disability from traumatic brain injury in the civilian population of the United States, 2005. J Head Trauma Rehabil. 2008;23(6):394-400.

3. Taylor CA, Bell JM, Breiding MJ, Xu L. Traumatic brain injury-related emergency department visits, hospitalizations, and deaths - United States, 2007 and 2013. MMWR Surveill Summ. 2017;66(9):1-16.

4. Majdan M, Plancikova D, Brazinova A, et al. Epidemiology of traumatic brain injuries in Europe: a cross-sectional analysis. Lancet Public Health. 2016;1(2):e76-e83.

5. Huang AR, Mallet L, Rochefort CM, Eguale T, Buckeridge DL, Tamblyn R. Medication-related falls in the elderly: causative factors and preventive strategies. Drugs Aging. 2012;29(5):359-376.

6. Deandrea S, Lucenteforte E, Bravi F, Foschi R, La Vecchia C, Negri E. Risk factors for falls in community-dwelling older people: a systematic review and meta-analysis. Epidemiology. 2010;21(5):658-668.

7. Park H, Satoh H, Miki A, Urushihara H, Sawada Y. Medications associated with falls in older people: systematic review of publications from a recent 5-year period. Eur J Clin Pharmacol. 2015;71(12):1429-1440.

8. Abbing-Karahagopian V, Huerta C, Souverein PC, et al. Antidepressant prescribing in five European countries: application of common definitions to assess the prevalence, clinical observations, and methodological implications. Eur J Clin Pharmacol. 2014;70(7):849-857.

9. Moore TJ, Mattison DR. Adult utilization of psychiatric drugs and differences by sex, age, and race. JAMA Intern Med. 2017;177(2):274-275.

10. Marcum ZA, Perera S, Thorpe JM, et al. Antidepressant use and recurrent falls in community-dwelling older adults: findings from the health ABC study. Ann Pharmacother. 2016;50(7):525-533.

11. Gribbin J, Hubbard R, Gladman J, Smith C, Lewis S. Serotonin-norepinephrine reuptake inhibitor antidepressants and the risk of falls in older people: case-control and case-series analysis of a large UK primary care database. Drugs Aging. 2011;28(11):895-902.

12. Naples JG, Kotlarczyk MP, Perera S, Greenspan SL, Hanlon JT. Nontricyclic and non-selective serotonin reuptake inhibitor antidepressants and recurrent falls in frail older women. Am J Geriatr Psychiatry. 2016;24(12):1221-1227. 
13. Ray WA, Griffin MR, Malcolm E. Cyclic antidepressants and the risk of hip fracture. Arch Intern Med. 1991;151(4):754-756.

14. Liu B, Anderson G, Mittmann N, To T, Axcell T, Shear N. Use of selective serotonin-reuptake inhibitors or tricyclic antidepressants and risk of hip fractures in elderly people. Lancet. 1998;351(9112):1303-1307.

15. Hubbard R, Farrington P, Smith C, Smeeth L, Tattersfield A. Exposure to tricyclic and selective serotonin reuptake inhibitor antidepressants and the risk of hip fracture. Am J Epidemiol. 2003;158(1):77-84.

16. Souverein PC, Abbing-Karahagopian V, Martin E, et al. Understanding inconsistency in the results from observational pharmacoepidemiological studies: the case of antidepressant use and risk of hip/femur fractures. Pharmacoepidemiol Drug Saf. 2016;25(Suppl 1):88-102.

17. Bakken MS, Engeland A, Engesæter LB, Ranhoff AH, Hunskaar S, Ruths $\mathrm{S}$. Increased risk of hip fracture among older people using antidepressant drugs: data from the Norwegian Prescription Database and the Norwegian Hip Fracture Registry. Age Ageing. 2013;42(4):514-520.

18. Aronson JK, editor. Meyler's Side Effects of Drugs. The International Encyclopedia of Adverse Drug Reactions and Interactions. 16th ed. Amsterdam: Elsevier Science; 2015.

19. Taipale H, Koponen M, Tanskanen A, et al. Risk of head and traumatic brain injuries associated with antidepressant use among communitydwelling persons with Alzheimer's disease: a nationwide matched cohort study. Alzheimers Res Ther. 2017;9(1):59.

20. van Doorn C, Gruber-Baldini AL, Zimmerman S, et al. Dementia as a risk factor for falls and fall injuries among nursing home residents. $J$ Am Geriatr Soc. 2003;51(9):1213-1218.

21. Tolppanen AM, Lavikainen P, Soininen H, Hartikainen S. Incident hip fractures among community dwelling persons with Alzheimer's disease in a Finnish nationwide register-based cohort. PLoS One. 2013;8(3):e59124.

22. Eom CS, Lee HK, Ye S, Park SM, Cho KH. Use of selective serotonin reuptake inhibitors and risk of fracture: a systematic review and metaanalysis. J Bone Miner Res. 2012;27(5):1186-1195.

23. Wu Q, Bencaz AF, Hentz JG, Crowell MD. Selective serotonin reuptake inhibitor treatment and risk of fractures: a meta-analysis of cohort and case-control studies. Osteoporos Int. 2012;23(1):365-375.

24. Rabenda V, Nicolet $\mathrm{D}$, Beaudart $\mathrm{C}$, Bruyère $\mathrm{O}$, Reginster JY. Relationship between use of antidepressants and risk of fractures: a metaanalysis. Osteoporos Int. 2013;24(1):121-137.

25. Coupland C, Dhiman P, Morriss R, Arthur A, Barton G, Hippisley-Cox J. Antidepressant use and risk of adverse outcomes in older people: population based cohort study. BMJ. 2011;343:d4551.

26. Pigeot I, Ahrens W. Establishment of a pharmacoepidemiological database in Germany: methodological potential, scientific value and practical limitations. Pharmacoepidemiol Drug Saf. 2008;17(3):215-223.

27. Schink T, Garbe E. Representativity of dispensations of non-steroidal antiinflammatory drugs (NSAIDs) in the German pharmacoepidemiological research database. Pharmacoepidemiol Drug Saf. 2010;19:S294-S94.

28. Ohlmeier C, Langner I, Garbe E, Riedel O. Validating mortality in the German Pharmacoepidemiological Research Database (GePaRD) against a mortality registry. Pharmacoepidemiol Drug Saf. 2016;25(7):778-784.

29. Jobski K, Schmedt N, Kollhorst B, Krappweis J, Schink T, Garbe E. Characteristics and drug use patterns of older antidepressant initiators in Germany. Eur J Clin Pharmacol. 2017;73(1):105-113.
30. Schmedt N, Jobski K, Kollhorst B, et al. Treatment patterns and characteristics of older antipsychotic users in Germany. Int Clin Psychopharmacol. 2016;31(3):159-169.

31. Schmedt N, Garbe E. Antipsychotic drug use and the risk of venous thromboembolism in elderly patients with dementia. J Clin Psychopharmacol. 2013;33(6):753-758.

32. Essebag V, Platt RW, Abrahamowicz M, Pilote L. Comparison of nested case-control and survival analysis methodologies for analysis of timedependent exposure. BMC Med Res Methodol. 2005;5(1):55.

33. Rothman KJ, Greenland S, Lash TL. Case-control studies. In: Rothman KJ, Greenland S, Lash TL, editors. Modern Epidemiology. 3rd ed. Philadelphia: Lippincott Williams \& Wilkins; 2008:111-27.

34. Ray WA. Evaluating medication effects outside of clinical trials: newuser designs. Am J Epidemiol. 2003;158(9):915-920.

35. Gardarsdottir H, Souverein PC, Egberts TC, Heerdink ER. Construction of drug treatment episodes from drug-dispensing histories is influenced by the gap length. J Clin Epidemiol. 2010;63(4):422-427.

36. Schneeweiss S, Avorn J. A review of uses of health care utilization databases for epidemiologic research on therapeutics. J Clin Epidemiol. 2005;58(4):323-337.

37. Mcconeghy KW, LeeY, Zullo AR, et al. Influenza illness and hip fracture hospitalizations in nursing home residents: are they related? J Gerontol A Biol Sci Med Sci. 2018;73(12):1638-1642.

38. Noguchi N, Chan L, Cumming RG, Blyth FM, Naganathan V. A systematic review of the association between lower urinary tract symptoms and falls, injuries, and fractures in community-dwelling older men. Aging Male. 2016;19(3):168-174.

39. Quan H, Li B, Couris CM, et al. Updating and validating the Charlson comorbidity index and score for risk adjustment in hospital discharge abstracts using data from 6 countries. Am J Epidemiol. 2011;173(6): 676-682.

40. Oderda LH, Young JR, Asche CV, Pepper GA. Psychotropic-related hip fractures: meta-analysis of first-generation and second-generation antidepressant and antipsychotic drugs. Ann Pharmacother. 2012;46(7-8):917-928.

41. Carrière I, Farré A, Norton J, et al. Patterns of selective serotonin reuptake inhibitor use and risk of falls and fractures in community-dwelling elderly people: the Three-City cohort. Osteoporos Int. 2016;27(11): 3187-3195.

42. Firsching ER, Mauer UM, Sakowitz OW, et al. Leitlinie Schädel-HirnTrauma im Erwachsenenalter (Guidelines on traumatic brain injuries in the adults). Jena: Deutsche Gesellschaft für Neurochirurgie DGNC; 2015.

43. Cunningham J, Williamson D, Robinson KM, Carroll R, Buchanan R, Paul L. The quality of medical record documentation and external cause of fall injury coding in a tertiary teaching hospital. Health Inf Manag. 2014;43(1):6-15.

44. Gagne JJ, Fireman B, Ryan PB, et al. Design considerations in an active medical product safety monitoring system. Pharmacoepidemiol Drug Saf. 2012;21(Suppl 1):32-40.

45. Maclure M, Fireman B, Nelson JC, et al. When should case-only designs be used for safety monitoring of medical products? Pharmacoepidemiol Drug Saf. 2012;21(Suppl 1):50-61.
Clinical Epidemiology

\section{Publish your work in this journal}

Clinical Epidemiology is an international, peer-reviewed, open access, online journal focusing on disease and drug epidemiology, identification of risk factors and screening procedures to develop optimal preventative initiatives and programs. Specific topics include: diagnosis, prognosis, treatment, screening, prevention, risk factor modification, Submit your manuscript here: https://www.dovepress.com/clinical-epidemiology-journa

\section{Dovepress}

systematic reviews, risk and safety of medical interventions, epidemiology and biostatistical methods, and evaluation of guidelines, translational medicine, health policies and economic evaluations. The manuscript management system is completely online and includes a very quick and fair peer-review system, which is all easy to use. 